\title{
Annotation-Based Empirical Performance Tuning Using Orio
}

\author{
Albert Hartono ${ }^{1}$, Boyana Norris ${ }^{2}$, and P. Sadayappan ${ }^{1}$ \\ ${ }^{1}$ Ohio State University, Dept. of Computer Science and Engineering, Columbus, OH 43210 \\ \{hartonoa, saday\}@cse.ohio-state.edu \\ ${ }^{2}$ Argonne National Laboratory, Mathematics and Computer Science Division, Argonne, IL 60439 \\ norris@mes.anl.gov
}

\begin{abstract}
In many scientific applications, significant time is spent tuning codes for a particular highperformance architecture. Tuning approaches range from the relatively nonintrusive (e.g., by using compiler options) to extensive code modifications that attempt to exploit specific architecture features. Intrusive techniques often result in code changes that are not easily reversible, which can negatively impact readability, maintainability, and performance on different architectures. We introduce an extensible annotation-based empirical tuning system called Orio, which is aimed at improving both performance and productivity by enabling software developers to insert annotations in the form of structured comments into their source code that trigger a number of low-level performance optimizations on a specified code fragment. To maximize the performance tuning opportunities, we have designed the annotation processing infrastructure to support both architecture-independent and architecture-specific code optimizations. Given the annotated code as input, Orio generates many tuned versions of the same operation and empirically evaluates the versions to select the best performing one for production use. We have also enabled the use of the PLuTo automatic parallelization tool in conjunction with Orio to generate efficient OpenMP-based parallel code. We describe our experimental results involving a number of computational kernels, including dense array and sparse matrix operations.
\end{abstract}

\section{Motivation}

The size and complexity of scientific computations are increasing at least as fast as the improvements in processor technology. Programming such scientific applications is hard, and optimizing them for high performance is even harder. This situation results in a potentially large gap between the achieved performance of applications and the peak available performance, with many applications achieving $10 \%$ or less of the peak (see, e.g., [4]). A greater concern is the inability of existing languages, compilers, and systems to deliver the available performance for the application through fully automated code optimizations.

Delivering performance without degrading productivity is crucial for the success of scientific computing. Scientific code developers generally attempt to improve performance by applying one or more of the following three approaches: manually optimizing code fragments; using tuned libraries for key numerical algorithms; and, less frequently, using compiler-based source transformation tools for loop-level optimizations. Manual tuning is time-consuming and impedes readability 
and performance portability. Tuned libraries often deliver great performance without requiring significant programming effort, but then can provide only limited functionality. General-purpose source transformation tools for performance optimizations are few and have not yet gained popularity among computational scientists, at least in part because of poor portability and steep learning curves.

\section{Related Work}

Ideally, a developer should have to specify only a few simple command-line options and then rely on the compiler to optimize the performance of an application on any architecture. Compilers alone, however, cannot fully satisfy the performance needs of scientific applications, for at least three reasons. First, compilers must operate in a black-box fashion and at a very low level, limiting both the type and number of optimizations that can be done. Second, static analysis of general-purpose languages, such as $\mathrm{C}, \mathrm{C}++$, and Fortran, is necessarily conservative, thereby precluding many possible optimizations. Third, extensive manual tuning of a code may prevent certain compiler optimizations and result in worse performance on new architectures, causing loss of performance portability.

An alternative to manual or automated tuning of application codes is the use of tuned libraries. The two basic approaches to supplying high-performance libraries involve providing a library of hand-coded options (e.g., [7, 8, 10]) and generating optimized code automatically for the given problem and machine parameters. ATLAS [25] for a reduced set of LAPACK routines, OSKI [23] for sparse linear algebra, PHiPAC [2] for matrix-matrix products, and domain-specific libraries such as FFTW [9] and SPIRAL [20] are all examples of the latter approach. Most automatic tuning approaches perform empirical parameter searches on the target platform. These automatic or handtuned approaches can deliver performance that can be five times as fast as that produced by many optimizing compilers [26]. The library approach, however, is limited by the fact that optimizations are highly problem- and machine-dependent. Furthermore, at this time, the functionality of the automated tuning systems is quite limited.

General-purpose tools for optimizing loop performance are also available. LoopTool [17] supports annotation-based loop fusion, unroll/jamming, skewing, and tiling. The Matrix Template Library [22] uses template metaprograms to tile at both the register and cache levels. A new tool, POET [27], also supports a number of loop transformations. Other research efforts whose goal, at least in part, is to enable optimizations of source code to be augmented with performancerelated information include the $\mathrm{X}$ language [6] (a macro $\mathrm{C}$-like language for annotating $\mathrm{C}$ code), the Broadway [16] compiler, and telescoping languages [12].

\section{Orio Design and Implementation}

Orio [18] is an empirical performance-tuning system that takes annotated C source code as input, generates many optimized code variants of the annotated code, and empirically evaluates the performance of the generated codes, ultimately selecting the best-performing version to use for production runs. Orio also supports automated validation by comparing the numerical results of the multiple transformed versions. 


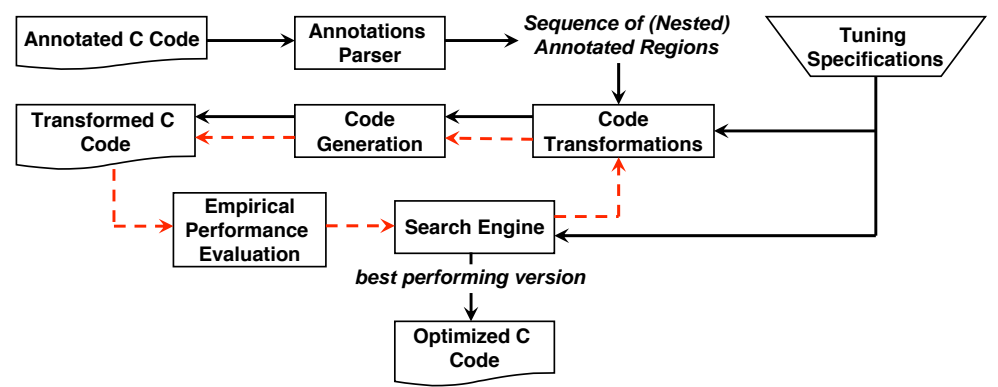

Figure 1: Overview of Orio's code generation and empirical tuning process.

The Orio annotation approach differs from existing compiler- and annotation-based systems in the following significant ways. First, through designing an extensible annotation parsing architecture, we are not committing to a single general-purpose language. Thus, we can define annotation grammars that restrict the original syntax, enabling more effective performance transformations (e.g., disallowing pointer arithmetic in a C-like annotation language); furthermore, it enables the definition of new high-level languages that retain domain-specific information normally lost in low-level $\mathrm{C}$ or Fortran implementations. This feature in turn expands the range of possible performance-improving transformations. Second, Orio was conceived and designed with the following requirements in mind: portability (which precludes extensive dependencies on external packages), extensibility (new functionality must require little or no change to the existing Orio implementation, and interfaces that enable integration with other source transformation tools must be defined), and automation (ultimately Orio should provide tools that manag all the steps of the performance tuning process, automating each step as much as possible). Third, Orio is usable in real scientific applications without requiring reimplementation. This ensures that the significant investment in the development of complex scientific codes is leveraged to the greatest extent possible.

Figure 1 shows a high-level graphical depiction of the code generation and tuning process implemented in Orio. Orio can be used to improve performance by source-to-source transformations such as loop unrolling, loop tiling, and loop permutation. The input to Orio is $\mathrm{C}$ code containing structured comments that include a simplified expression of the computation, as well as various performance-tuning directives. Orio scans the marked-up input code and extracts all annotation regions. Each annotation region is then processed by transformation modules. The code generator produces the final $\mathrm{C}$ code with various optimizations that correspond to the specified annotations. Unlike compiler approaches, we do not implement a full-blown C compiler; rather, we use a precompiler that parses only the language-independent annotations.

Orio can also be used as an automatic performance-tuning tool. The code transformation modules and code generator produce an optimized code version for each distinct combination of performance parameter values. Then each optimized code version is executed and its performance evaluated. After iteratively evaluating all code variants, the best-performing code is picked as the final output of Orio. Because the search space of all possible optimized code versions can be huge, a brute-force search strategy is not always feasible. Hence, Orio provides various search heuristics for reducing the size of the search space and thus the empirical testing time. 


\subsection{Annotation Language Syntax}

Orio annotations are embedded into the source code as structured $\mathrm{C}$ comments that always start with $/ * a$ and end with $a * /$. For example, the annotation $/ * a$ end $a * /$ is used to indicate the end of an annotated code region. A simple grammar illustrating the basic syntax of Orio annotations is depicted in Figure 2. An annotation region consists of three main parts: leader annotation, annotation body, and trailer annotation. The annotation body can either be empty or contain $\mathrm{C}$ code that possibly includes other nested annotation regions. A leader annotation contains the name of the code transformation module used to transform and generate the annotated application code. A high-level description of the computation and several performance hints are coded in the module body inside the leader annotation. A trailer annotation, which has a fixed form (i.e., /*@ end $@ * /$ ), closes an annotation region.

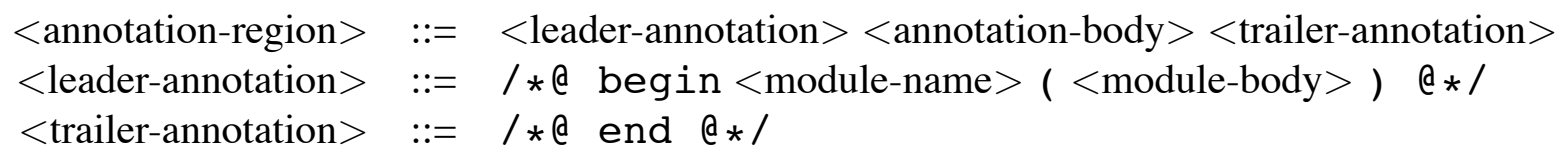

Figure 2: Annotation language grammar excerpt.

\subsection{Orio Input Example}

Figure 3 shows a concrete annotation example that empirically optimizes a $\mathrm{C}$ function for the Blue Gene/P architecture. This is an instance of an AXPY operation, one that computes $y=$ $y+a_{1} x_{1}+\cdots+a_{n} x_{n}$, where $a_{1}, \ldots, a_{n}$ are scalars and $y, x_{1}, \ldots, x_{n}$ are one-dimensional arrays. The specific AXPY operation considered in this example corresponds to $n=4$. The first annotation contains the BGP_Align ${ }^{1}$ directive, which instructs Orio to dynamically load its memoryalignment optimization module and then generate preprocessor directives, such as pragmas and calls to memory alignment intrinsics, including a check for data alignment. The main purpose of these alignment optimizations is to enable the use of the dual floating-point unit (Double Hummer) of the Blue Gene/P, which requires 16-byte alignment. As discussed later in Section 5.1, even these simple alignment optimizations can lead to potentially significant performance improvements. This example also shows the use of Orio's loop transformation module (named Loop) to optimize the AXPY-4 loop by unrolling and generating OpenMP parallelization directives for exploiting multicore parallelism. In addition to the simple source transformations in this example, Orio supports other optimizations, such as register tiling and scalar replacement.

Whereas the BGP_Align and Loop annotations in this example guide the source-to-source transformations, the purpose of the PerfTuning annotation is primarily to control the empirical performance-tuning process. Details of the tuning specifications for optimizing the AXPY-4 code on the Blue Gene/P are shown in the right-hand side of Figure 3. The tuning specification contains data required for building, initializing, and running experiments, including input variable information, the search algorithm, performance counting technique, performance parameters values, and

\footnotetext{
${ }^{1}$ Architecture-specific annotations are simply ignored when the code is being tuned on an architecture that doesn't support them.
} 
cation of the existing Orio software. Therefore, varied approaches to code transformations ranging from low-level loop optimizations for cache performance to composed linear algebra operations and new specialized algorithms can easily be integrated into Orio.

After parsing the annotation, each module performs a distinct optimization transformation prior to generating the optimized code. The transformation module can either reuse an existing annotation parser or define new language syntax and a corresponding parser component.

In some cases, the annotation is seemingly redundant, containing a version of the computation very similar to the original code. As mentioned earlier, we took this approach so that the annotation language can be defined in a way that enables more effective transformations (through restrictions or high-level domain information).

Current optimizations supported by Orio span different types of code transformations that are not provided by production compilers in some cases. Available optimizations include simple loop unrolling, memory alignment optimization, loop unroll/jamming, loop tiling, loop permutation, scalar replacement, register tiling, loop-bound replacement, array copy optimization, multicore parallelization (using OpenMP), and other architecture-specific optimizations (e.g., generating calls to SIMD intrinsics on Intel and Blue Gene/P architectures).

\subsection{Search Space Exploration and Evaluation}

As briefly discussed earlier, our empirical tuning approach systematically measures the performance costs of automatically generated code variants in order to find the most optimal available version. In the context of empirical optimization, code variants are alternative, semantically equivalent, implementations of the same computation. Each implementation variant is associated with a collection of different optimization parameters that correspond to source-to-source code transformations such as unroll factors, tile sizes, and loop permutation order. Hence, each coordinate in the search space of empirical tuning problem represents a distinct combination of performance parameter values. The dimension and the size of the search space depend on the total number and the value ranges of used performance parameters, respectively.

The conceptually straightforward approach to exploring the space of the parameter values is to use an exhaustive search procedure that is guaranteed to determine the optimal code version. Normally, however, the size of the search space is too large, making full coverage impractical. Thus, in addition to supporting exhaustive search, we have implemented several search heuristics. The simplest search heuristic is a random search, which picks a random coordinate in the search space at each step and then measures its performance; random search is not guaranteed to return close-to-optimal results. We have also developed two other search heuristics to effectively narrow the search space for close-to-optimal performance: a heuristic based on the Nelder-Mead simplex method $[14,15]$, a popular non-derivative direct search method for optimization, and simulated annealing [13]. Similarly to the implementation of Orio's optimization modules, each search technique is implemented as an independent Python module in Orio and is dynamically loaded given only the algorithm's name as one of the fields in the tuning specification.

In order to improve the quality of the search result further, each search heuristic is enhanced by applying a local search after the global search completes. The local search compares the best performance with neighboring coordinates. If a better coordinate is discovered, the local search continues recursively until no further performance improvement is possible or a user-specified termination criterion is met. 


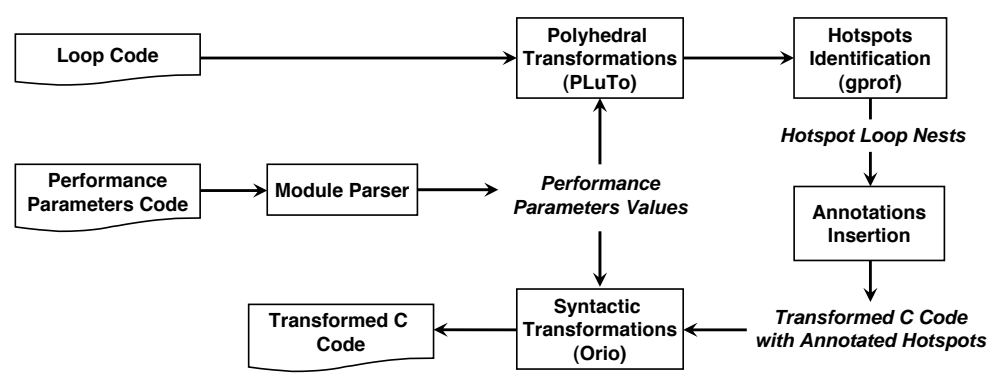

Figure 5: Integration of PLuTo into Orio's code transformation module.

called PLuTo [3]. This integration also demonstrates the easy extensibility of Orio and the ability to leverage other source transformation approaches.

PLuTo is a source-to-source automatic transformation tool aimed at optimizing a sequence of nested loops for data locality and coarse-grained parallelism simultaneously. PLuTo employs a polyhedral model of arbitrary loop nests, where the dynamic instance (iteration) of each statement is viewed as an integer point in a well-defined space called the statement's polyhedron. This statement representation and a precise characterization of data dependences enable PLuTo to construct mathematically correct complex loop transformations. PLuTo's polyhedral-based transformations result in improved cache locality and loops parallelized for multicore architecture.

Figure 5 outlines the overall structure of the PLuTo-Orio integrated system, which is implemented as a new optimization module in Orio. In addition to the loop code to be optimized, the module parses performance parameters, which include tile sizes, unroll factors, and loop order (permutation), as well as several Boolean values for triggering OpenMP parallelization, scalar replacement, and autovectorization. Using these parameter values, PLuTo then performs polyhedral transformations (e.g., two-level tiling and OpenMP parallelization) on the input loop code. The resulting generated code is subsequently passed to the widely available profiling tool gprof [11] for hotspot detection. The identified hotspot loop nests are then marked with Orio's performance annotations for complementary syntactic transformations (e.g., loop permutation, loop unroll/jamming, scalar replacement, and explicit autovectorization). Finally, Orio optimizes the annotated hotspots as described in Section 3.

PLuTo also performs syntactic loop unroll/jamming as a postprocessing pass. The target loops, however, are limited to innermost loops with a maximum depth of two (i.e., 1-D unrolling and 2-D unroll/jamming). We therefore choose to use the loop unroll/jamming transformation already available in Orio, which is more flexible because it can be applied to loop nests of depths larger than two.

At present Orio does not employ any data dependency analysis when performing syntactic transformations. Therefore, there is no guarantee that the code generated after syntactic transformations is correct. Hence, the tuning process using the integrated PLuTo-Orio system is currently semi-automatic-user involvement is required to decide whether it is safe to apply a syntactic transformation on an identified hotspot. 


\section{Experimental Results}

In this section, we discuss the performance results of several experiments on a multicore Intel Xeon workstation and a Blue Gene/P (both at Argonne). The workstation has dual quad-core E5462 Xeon processors (8 cores total) running at $2.8 \mathrm{GHz}(1600 \mathrm{MHz}$ FSB) with $32 \mathrm{~KB}$ L1 cache, $12 \mathrm{MB}$ of L2 cache (6 MB shared per core pair), and 2 GB of DDR2 FBDIMM RAM, running Linux kernel version 2.6.25 (x86-64). Each compute node of the Blue Gene/P is equipped with four $850 \mathrm{MHz}$ IBM PowerPC 450 processors with a dual floating-point unit and $2 \mathrm{~GB}$ total memory per node, private L1 (32 KB) and L2 (4 MB) caches, a shared L3 cache (8 MB), and running a proprietary lightweight operating system. We used version 10.1 of the Intel and version 9.0 of the IBM XL C/C++ V9.0 compilers on the Xeon and Blue Gene/P, respectively. Because of space considerations, here we discuss a limited number of computational kernels; more performance results for different computations are available at the Orio project website [18].

\subsection{Sequence of Linear Algebra Operations}

In this experiment, we tuned the performance of the AXPY-4 operation (see Figure 3) on a single node of the Blue Gene/P machine using the IBM xlc compiler. We measured the performance for two scenarios: using a single core per node and using all four cores. The results are shown in Figures 6(a) and 6(b), respectively. The single-core scenario was compiled with the following options: -03 -qstrict -qarch $=450 \mathrm{~d}$-qtune $=450$-qhot -qsmp=noauto; the multicore scenario differs in the use of -qsmp=auto for the non-Orio versions. The parallel Orio version contains OpenMP parallelization directives in the generated code, thus necessitating the use of the -qsmp=omp : noauto compiler option. Included are performance numbers for four code variants: a simple loop implementation without any library calls (labeled "Compiler-optimized"), two BLAS-based implementations that use Goto BLAS [10] and the ESSL [8] libraries, and the Orio-tuned version.

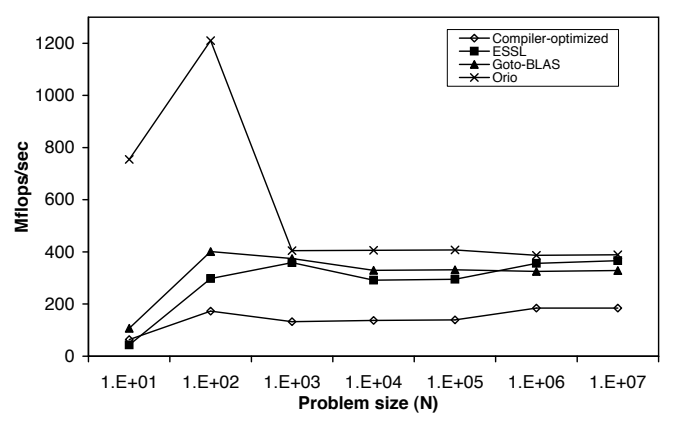

(a) Sequential (single core)

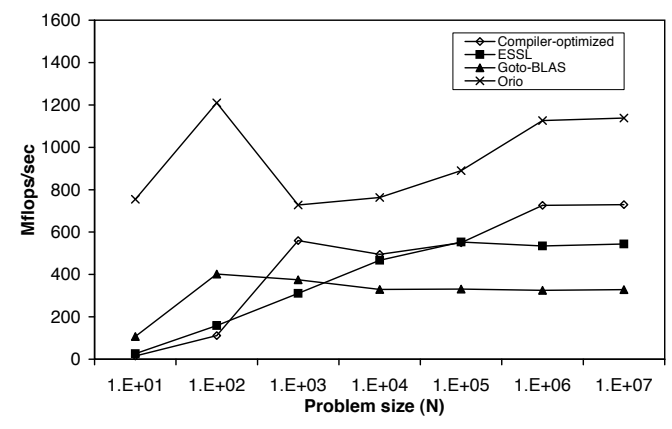

(b) Parallel (four cores)

Figure 6: Performance of AXPY-4 operations on the Blue Gene/P.

The performance results shown in Figure 6 indicate that the code tuned by Orio consistently outperforms the other three versions for both the sequential and parallel cases. We observe that even for a simple algebraic operation, such as the composed AXPY routines, the compiler alone is unable to yield performance comparable to the empirically tuned version. Moreover, implementations that rely on calls to multiple tuned library routines (e.g., Goto BLAS and ESSL) suffer from 


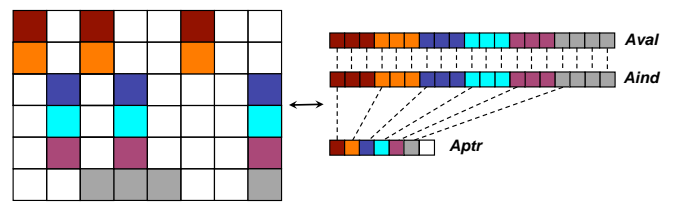

(a)

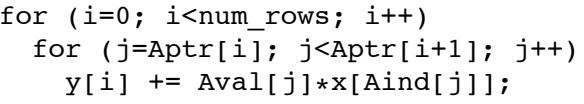

(b)

Figure 7: (a) Compressed sparse row (CSR) format; (b) Basic implementation of CSR-based SpMV.

loss of both spatial and temporal localities, resulting in inferior memory performance.

\subsection{Sparse Matrix Computations}

In this section we examine the effectiveness of Orio in optimizing key computations in PETSc [1], a toolkit for the parallel numerical solution of partial differential equations, by empirically tuning one of its heavily used kernels, sparse matrix-vector multiplication (SpMV). SpMV dominates the performance of various scientific applications; yet, traditional implementations of sparse kernels exhibit relatively poor performance because of the use of indirect addressing for accessing the matrix data elements.

The SpMV operation computes $\forall_{A_{i, j}} \neq 0: y_{i} \leftarrow y_{i}+A_{i, j} \cdot x_{j}$, where $A$ is a sparse matrix, and $x, y$ are dense vectors. Each element of $A$ is used precisely once, and element reuse is possible only for $x$ and $y$. Thus, to optimize SpMV, one should use compact data structures for $A$ and try to maximize temporal reuse of $x$ and $y$. One of the most commonly used data structures for storing a sparse matrix is compressed sparse row (CSR) storage [24], illustrated in Figure 7(a). Elements in each row of $A$ are packed together in a dense array, Aval, and a corresponding array of integers Aind stores the column indices. The Aptr array designates where each sparse row begins in Aval and Aind. An implementation of SpMV using CSR storage is shown in Figure 7(b).

PETSc's implementation of SpMV used in this experiment exploits matrix structure by using inodes, which represent rows with identical nonzero structure. PETSc detects inodes automatically in arbitrary sparse matrices. For example, the sparse matrix shown in Figure 7(a) contains three inodes. The first inode is of size two because it holds two adjacent rows (i.e., the first and the second rows) with the same nonzero structure. The second inode contains three consecutive rows with identical nonzero structure, and the last inode has only a single row. Knowing the properties of each inode at runtime enables maximum reuse of vector $x$ since multiple elements of $x$ can be loaded and used exactly once for all rows in the same inode structure. To accomplish this, one must use a register-blocking transformation. To optimize the inode-based SpMV routine, we incorporated a new transformation module inside Orio that implements various optimization strategies including register blocking, SIMDization, memory alignment optimization, loop-control optimization, accumulator expansion, and thread-level parallelization (with OpenMP). We then used Orio to automatically select the best version of the optimized inode SpMV. Only the outer loop that iterates over inodes was parallelized by using OpenMP.

To evaluate the performance of the tuned SpMV routine, we conducted the experiment using a 2-D driven cavity flow simulation application [5] (SNES ex27 in PETSc), on both the Xeon and Blue Gene/P. The "Compiler-optimized" label is used as a base case that represents the performance of the naive implementation of SpMV (shown in Figure 7(b)) optimized by the native compiler. We also tested the performance of the hand-tuned (by PETSc developers) SpMV code, 
which is included in PETSc releases.

Figure 8 shows the performance results on the Intel Xeon. The problem size is the number of grid points in the $x$ and $y$ directions, not the size of the sparse input matrix. The matrix dimension is based on the grid size and the number of field components computed for each grid point; for example, an $8 \times 8$ problem involves sparse matrices of dimension 900 with 17,040 nonzero entries. In this experiment, we ran in three multicore modes: SMP (one MPI process, eight threads/process), dual (four MPI processes, two threads/process), and virtual node or VN (eight MPI processes, one thread/process). The code tuned by Orio consistently outperforms the others. Furthermore, the performance of the hand-tuned code is almost equivalent to that of the icc-optimized simple code. Performance differences between the Orio version and the hand-tuned version become more significant as more threads per process are employed to facilitate OpenMP parallelization. The SMP and VN results show that optimizations using loop-level parallelism (through OpenMP) achieve much better performance than using MPI coarse-grained parallelism.

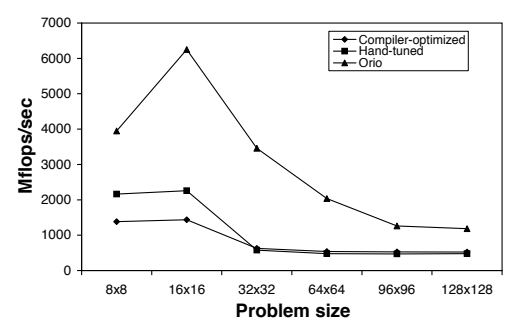

(a) SMP: $p=1, t / p=8$

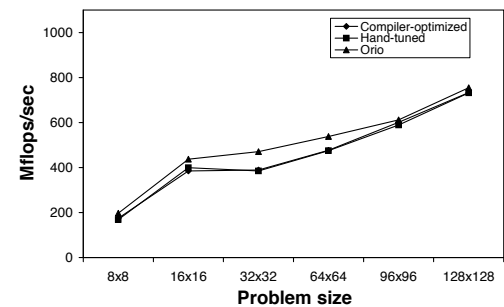

(b) Dual: $p=4, t / p=2$

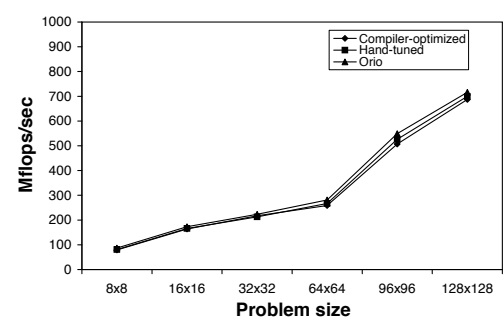

(c) VN: $p=8, t / p=1$

Figure 8: Performance of inode SpMV on eight-core Intel machine; $p$ is the number of processes, and $t / p$ is the threads per process.

The Blue Gene/P results in Figure 9 again show that empirical optimization using Orio produces the best performance for all cases. On this architecture, the performance gap between the xlc-optimized and the hand-tuned codes is now larger than in the Intel experiment. Similarly, by exploiting thread-level parallelism, the Orio-optimized code performs better than the hand-tuned version when the number of threads per process increases and the number of nodes decreases. For the single-thread cases (VN mode), thread-level parallelism is not exploited; nevertheless, the Orio version still performs better than the other two choices.

\subsection{Evaluation of the PLuTo-Orio Integrated System}

This section discusses the performance evaluation of the integrated PLuTo-Orio system (discussed in Section 4) on the multicore Intel Xeon by using a number of application kernels that are nontrivial to optimize and parallelize. We compare the performance of the code tuned by Orio with the base code and the PLuTo-generated codes. The PLuTo code was obtained by running the base code with PLuTo-0.3.0 [19] using --tile to employ loop tiling for L1 cache, --12tile to employ loop tiling for L2 cache, and --unroll to employ loop unrolling, and for parallel code generation, an additional --parallel option. We used PLuTo's default tile sizes (L1: 32x32

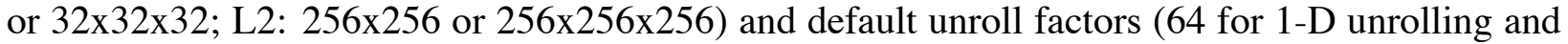
$8 \times 8$ for $2-\mathrm{D}$ unroll/jamming). All codes were compiled with the Intel $\mathrm{C}$ compiler using the -03 


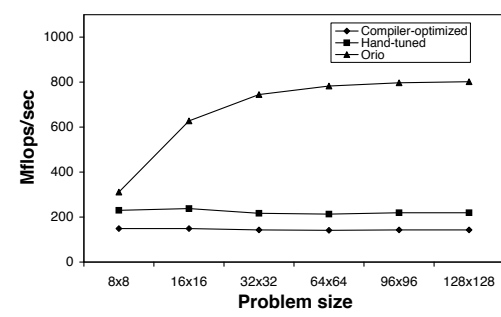

(a) 1 node, SMP mode

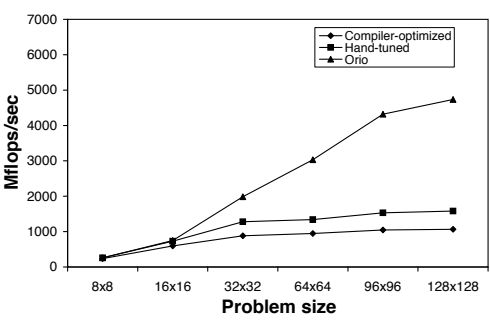

(d) 8 nodes, SMP mode

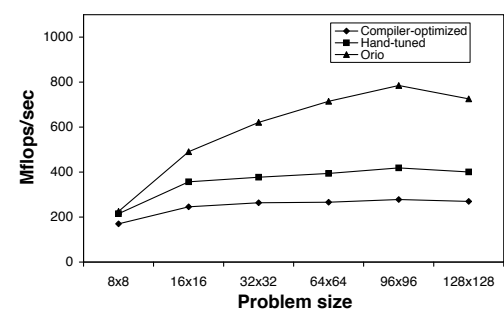

(b) 1 node, Dual mode

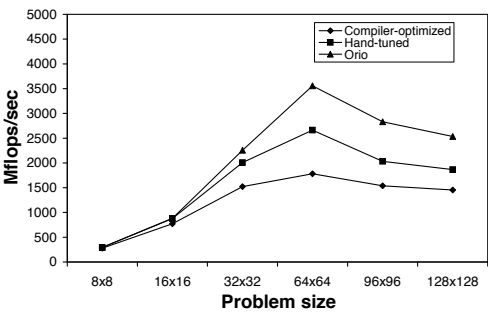

(e) 8 nodes, Dual mode

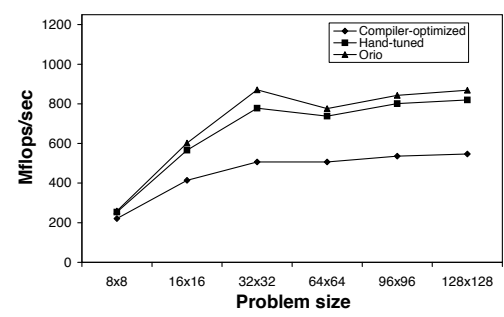

(c) 1 node, $\mathrm{VN}$ mode

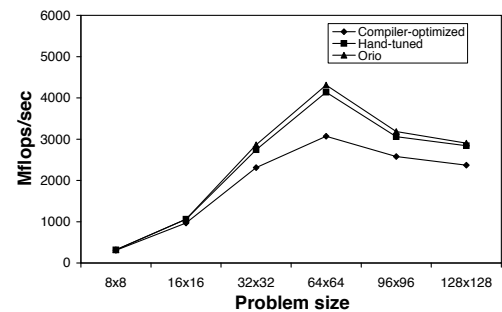

(f) 8 nodes, VN mode

Figure 9: Performance of inode SpMV on Blue Gene/P for 1 and 8 nodes.

optimization flag to enable auto-vectorization and other advanced optimizations, and -parallel (or -openmp in the case of manual OpenMP parallelization) to enable code parallelization.

In the following sections, we refer to the icc-optimized base code as "ICC," the PLuTo-generated code with L1 tiling and unroll/jamming as "PLuTo (L1 tiling)," and the PLuTo-generated code with L1 and L2 tilings and unroll/jamming as "PLuTo (L1+L2 tiling)." The code tuned by Orio is referred to as "PLuTo+Orio."

\subsubsection{2-D Finite-Difference Time-Domain Method for Computational Electromagnetics}

We consider the two-dimensional finite difference time domain (FDTD) algorithm, a popular method for solving the time-dependent Maxwell's equations in the context of computational electrodynamic problems. As shown in Figure 10, the 2-D FDTD method is implemented as an outer iteration over time containing four imperfectly nested loops. The arrays $e x$ and $e y$ denote the electric field components, and the array $h z$ denotes the magnetic field.

The performance of the sequential 2-D FDTD code for tmax $=500$ and $n x=n y$ is shown in Figure 11(a). The base code optimized by icc alone performs better than PLuTo for small problem sizes since all input arrays fit in the L2 cache

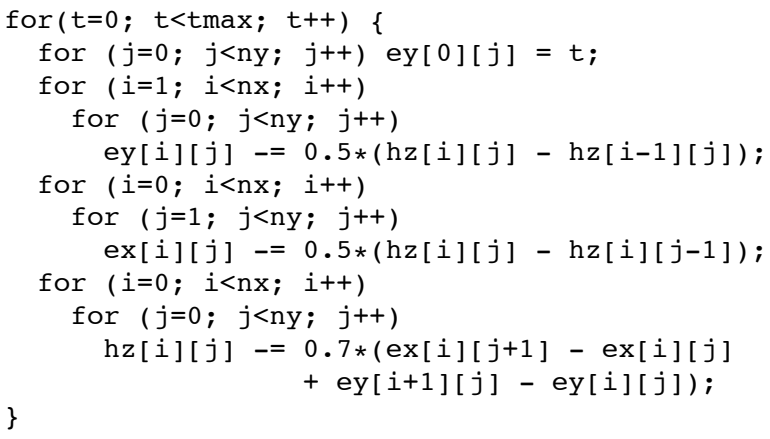

Figure 10: 2-D FDTD code. (insufficient computation to offset PLuTo's tiling overhead). As the array sizes increase, the lack of data reuse impairs the base code's performance, whereas the PLuTo performance remains about the same. When the input arrays are small, Orio discovers that applying PLuTo's polyhedral transformations is not beneficial, and therefore it employs only its syntactic transformations on the original FDTD code. For large problem sizes, Orio 
exploits some of the PLuTo's code transformations and enhances these further with its syntactic optimizations, resulting in performance consistently and significantly higher than both the base and PLuTo codes (up to $86 \%$ over PLuTo).

Figure 11(b) shows the multicore performance obtained for tmax $=500$ and $n x=n y=$ 2000. The results indicate that whereas icc is unable to autoparallelize the code, PLuTo detects the existence of pipelined parallelism and then successfully parallelizes the code. Locality-exploiting optimizations by Orio additionally improve the PLuTo performance by up to $78 \%$. Moreover, we observe that because of memory contention between the two quad-core Intel processors, the speedup of both the PLuTo and Orio codes slightly decreases when the number of cores used is greater than four.

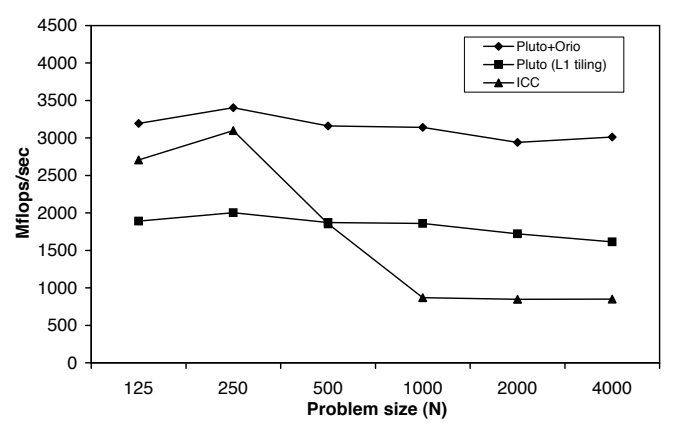

(a) Sequential $(\mathrm{T}=500)$

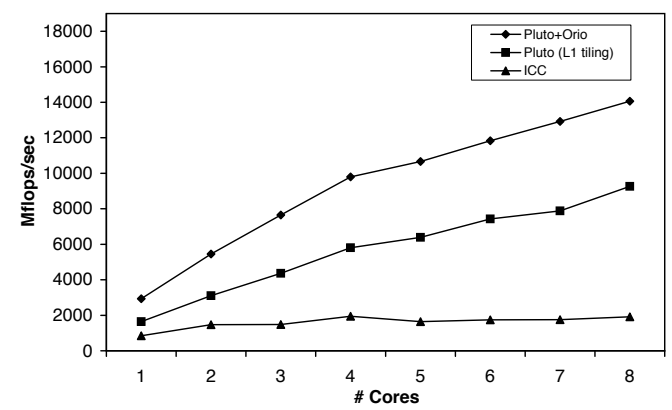

(b) Parallel $(\mathrm{T}=500, \mathrm{~N}=2000)$

Figure 11: 2-D FDTD performance on an eight-core Intel Xeon.

\subsubsection{3-D Gauss-Seidel Successive Overrelaxation Method}

Figure 12 shows the 3-D Gauss-Seidel computation, sometimes referred to as successive displacement method, indicating the dependence of the iterations on the ordering. If the ordering is changed, the components of the new iterations will change as well.

Figure 13(a) contains the sequential perforfor $(t=0 ; t<=T-1 ; t++)$ for $(i=1 ; i<=\mathrm{N}-2 ; i++)$ for $(j=1 ; j<=N-2 ; j++)$ $A[i][j]=(A[i-1][j-1]+A[i-1][j]$

$+A[i-1][j+1]+A[i][j-1]+A[i][j]$

$+A[i][j+1]+A[i+1][j-1]+A[i+1][j]$

$+A[i+1][j+1]) / 9.0$; ing PLuTo's polyhedral tiling on the original code always delivers performance boosts, which range from $126 \%$ to $142 \%$. When using PLuTo, perform-

Figure 12: 3-D Gauss-Seidel code ing two-level tiling (for both L1 and L2 caches) yields 13\% lower performance than performing only L1 tiling. This is also reflected in the best sequential code found by Orio, where one-level tiling (for L1 cache only) is always performed. The sequential speedup obtained from tuning the PLuTo code with Orio is significant, ranging from $51 \%$ to $133 \%$.

The parallel performance results for $T=500$ and $N=4000$ are shown in Figure 13(b). Again we observe that icc failed to parallelize the code, whereas PLuTo was able to parallelize it and Orio improved the performance of the one-level tiled PLuTo code further by up to 128\% (and up to $548 \%$ for the two-level tiled PLuTo code). We observed that tiling over both L1 and L2 can result in worse performance than L1-only tiling because the number of tiles can be smaller than the number of cores. 


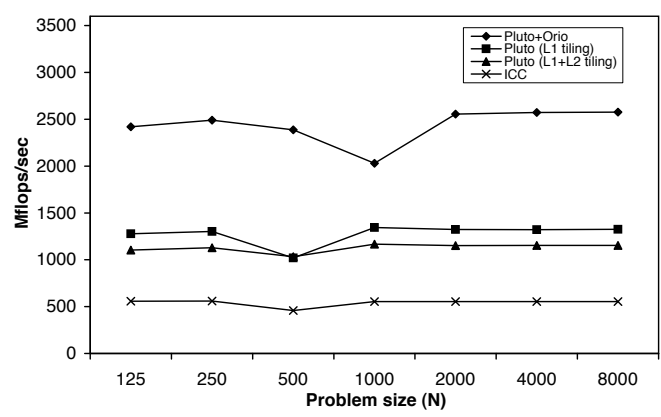

(a) Sequential $(\mathrm{T}=500)$

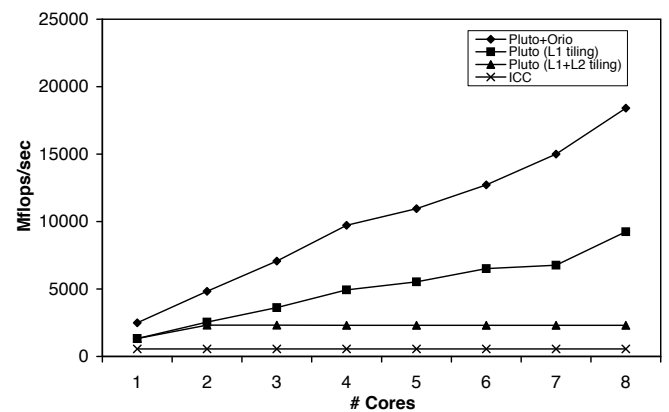

(b) Parallel $(\mathrm{T}=500, \mathrm{~N}=4000)$

Figure 13: 3-D Gauss-Seidel performance on eight-core Intel machine.

\subsubsection{LU Factorization}

LU factorization or decomposition is a numerical method for the solution of linear systems of equations; a simple implementation is shown in Figure 14.

The sequential performance results are shown in Figure 15(a). Similarly to the 2-D FDTD results, the icc-optimized code is more efficient than the PLuTo-generated codes when the input arrays are small and fit in the L2 cache. Because of bet-

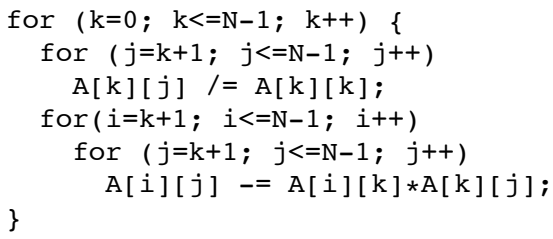

Figure 14: LU decomposition code. ter data locality, however, the performance of the PLuTo-tiled codes is better than that of the base code as the input arrays get larger. Consequently, Orio employs PLuTo's polyhedral transformations only for large input sizes prior to applying its own syntactic transformations. Compared to the two PLuTo codes, the Orio-tuned code yields performance improvements ranging between $26 \%$ and $277 \%$.

The parallel performance results shown in Figure 15(b) were obtained for $N=4000$. We observe that icc is not able to parallelize the code, whereas PLuTo achieves higher performance by exploiting multicore parallelism. Furthermore, both PLuTo-generated codes have comparable performance, with slightly better scalability exhibited by the single-level tiled code. Orio further improved the performance of the PLuTo-generated versions by a factor of $51 \%$ to $120 \%$.

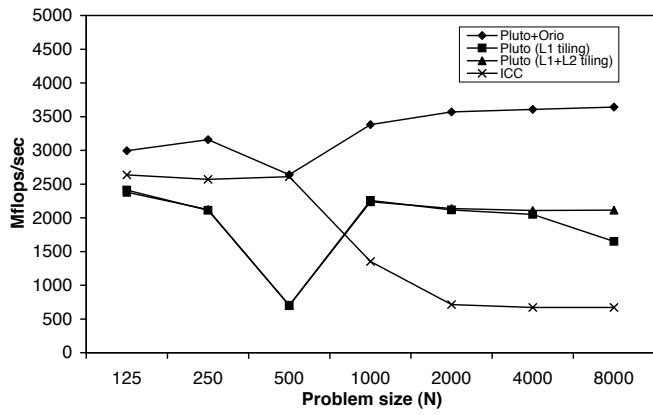

(a) Sequential

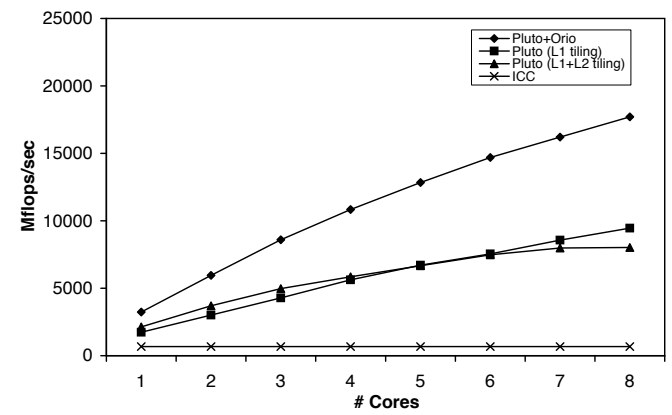

(b) Parallel (N=4000)

Figure 15: LU Decomposition performance on eight-core Intel machine. 


\section{Conclusions and Future Work}

We have described the design and implementation of Orio, an extensible Python software system for defining annotation-based performance-improving transformations. Our experiments with a number of different types of computations on two different architectures show that Orio can deliver performance improvements when used alone or in conjunction with other source transformation tools.

Orio is a new tool under active development; future work includes providing support for annotating and generating Fortran code, defining new annotation languages and corresponding transformation modules, (e.g., using matrix notation for linear algebra operations), and integrating with other source transformation tools through new optimization modules.

Acknowledgments. We would like to thank Uday Bondhugula of Ohio State University for many productive discussions and his valuable help with PLuTo. This work was supported in part by the U.S. Dept. of Energy under Contract DE-AC02-06CH11357.

\section{References}

[1] S. Balay, K. Buschelman, V. Eijkhout, W. D. Gropp, D. Kaushik, M. G. Knepley, L. C. McInnes, B. F. Smith, and H. Zhang. PETSc Users Manual. Technical Report ANL-95/11 - Revision 2.1.5, Argonne National Laboratory, 2004.

[2] J. Bilmes, K. Asanovic, C.-W. Chin, and J. Demmel. Optimizing matrix multiply using PHiPAC: A portable, high-performance, ANSI C coding methodology. In International Conference on Supercomputing, pages 340-347, 1997.

[3] U. Bondhugula, A. Hartono, J. Ramanujam, and P. Sadayappan. A practical automatic polyhedral program optimization system. In ACM SIGPLAN Conference on Programming Language Design and Implementation (PLDI), June 2008.

[4] J. Carter, L. Oliker, and J. Shalf. Performance evaluation of scientific applications on modern parallel vector systems. In M. J. Daydé, J. M. L. M. Palma, A. L. G. A. Coutinho, E. Pacitti, and J. C. Lopes, editors, VECPAR, volume 4395 of Lecture Notes in Computer Science, pages 490-503, Germany, 2006. Springer.

[5] T. S. Coffey, C. T. Kelley, and D. E. Keyes. Pseudo-transient continuation and differential-algebraic equations. SIAM J. Sci. Comput., 25(2):553-569, 2003.

[6] S. Donadio, J. Brodman, T. Roeder, K. Yotov, D. Barthou, A. Cohen, M. J. Garzarán, D. Padua, and K. Pingali. Language for the compact representation of multiple program versions. In Proceedings of Languages and Compilers for Parallel Computing (LCPCO5), volume 4339 of Lecture Notes in Computer Science, pages 136-151. Springer, Germany, 2006.

[7] J. J. Dongarra, J. D. Croz, I. S. Duff, and S. Hammarling. A set of level 3 basic linear algebra subprograms. ACM Trans. Math. Soft., 16:1-17, 1990.

[8] Engineering Scientific Subroutine Library (ESSL) and parallel ESSL. www-03.ibm.com/ systems/p/software/essl.html, 2006. 
[9] M. Frigo. FFTW: An adaptive software architecture for the FFT. In Proceedings of the ICASSP Conference, volume 3, page 1381, 1998.

[10] K. Goto and R. van de Geijn. High-performance implementation of the level-3 BLAS. Technical Report TR-2006-23, The University of Texas at Austin, Department of Computer Sciences, 2006.

[11] S. L. Graham, P. B. Kessler, and M. K. McKusick. Gprof: A call graph execution profiler. SIGPLAN Not., 39(4):49-57, 2004.

[12] K. Kennedy et al. Telescoping languages project description. telescoping.rice.edu, 2006.

[13] S. Kirkpatrick, C. D. Gelatt, and M. P. Vecchi. Optimization by simulated annealing. Science, 220:671680, 1983.

[14] J. C. Lagarias, J. A. Reeds, M. H. Wright, and P. E. Wright. Convergence properties of the NelderMead simplex method in low dimensions. SIAM J. Optimization, 9:112-147, 1998.

[15] R. M. Lewis, Michael, and W. Trosset. Direct search methods: Then and now. Journal of Computational and Applied Mathematics, 124:200-0, 2000.

[16] C. Lin and S. Z. Guyer. Broadway: A compiler for exploiting the domain-specific semantics of software libraries. Proceedings of the IEEE, 93(2):342-357, July 2005.

[17] J. Mellor-Crummey, R. Fowler, G. Marin, and N. Tallent. HPCVIEW: A tool for top-down analysis of node performance. The Journal of Supercomputing, 23(1):81-104, Aug 2002.

[18] Orio project. trac.mcs.anl.gov/projects/performance/orio, 2008.

[19] The PLuTo automatic parallelizer. sourceforge.net/projects/pluto-compiler, 2008.

[20] M. Puschel, J. M. F. Moura, J. R. Johnson, D. Padua, M. M. Veloso, B. W. Singer, J. Xiong, F. Franchetti, A. Gacic, Y. Voronenko, K. Chen, R. W. Johnson, and N. Rizzolo. SPIRAL: Code generation for DSP transforms. In Proceedings of the IEEE, Special Issue on Program Generation, Optimization, and Platform Adaptation, volume 93, pages 216-231, Feb. 2005.

[21] V. Sarkar. Optimized unrolling of nested loops. Int. J. Parallel Program., 29(5):545-581, 2001.

[22] J. G. Siek and A. Lumsdaine. A rational approach to portable high performance: The Basic Linear Algebra Instruction Set (BLAS) and the Fixed Algorithm Size Template (FAST) library. In Parallel Object Oriented Scientific Computing. ECOOP, 1998.

[23] R. Vuduc, J. Demmel, and K. Yelick. OSKI: A library of automatically tuned sparse matrix kernels. In Proceedings of SciDAC 2005, volume 16 of Journal of Physics: Conference Series, pages 521-530. Institute of Physics Publishing, June 2005.

[24] R. W. Vuduc. Automatic performance tuning of sparse matrix kernels. PhD thesis, 2003. Chair-James W. Demmel.

[25] R. C. Whaley and J. J. Dongarra. Automatically tuned linear algebra software. In Supercomputing '98: Proceedings of the 1998 ACM/IEEE Conference on Supercomputing (CDROM), pages 1-27. IEEE Computer Society, 1998.

[26] R. C. Whaley, A. Petitet, and J. J. Dongarra. Automated empirical optimization of software and the ATLAS project. Parallel Computing, 27(1-2):3-35, 2001. 
[27] Q. Yi, K. Seymour, H. You, R. Vuduc, and D. Quinlan. POET: Parameterized optimizations for empirical tuning. In Proceedings of the Parallel and Distributed Processing Symposium, 2007, pages 1-8. IEEE Computer Society, March 2007. 
The submitted manuscript has been created by UChicago Argonne, LLC, Operator of Argonne National Laboratory ("Argonne"). Argonne, a U.S. Department of Energy Office of Science laboratory, is operated under Contract No. DE-AC02-06CH11357. The U.S. Government retains for itself, and others acting on its behalf, a paid-up, nonexclusive, irrevocable worldwide license in said article to reproduce, prepare derivative works, distribute copies to the public, and perform publicly and display publicly, by or on behalf of the Government. 